\title{
Transformation of liver cells by 3-methylcholanthrene potentiates oxidative stress via the downregulation of glutathione synthesis
}

\author{
SOU HYUN KIM ${ }^{1 *}$, DAHYE YOON ${ }^{2 *}$, YUN-HEE LEE $^{3}$, JAEWON LEE $^{1}$, NAM DEUK KIM ${ }^{1}$, \\ SUHKMANN KIM ${ }^{2}$ and YOUNG-SUK JUNG ${ }^{1}$ \\ ${ }^{1}$ College of Pharmacy, Pusan National University; ${ }^{2}$ Department of Chemistry, Center for Proteome \\ Biophysics and Chemistry Institute for Functional Materials, Pusan National University, Busan 46241; \\ ${ }^{3}$ College of Pharmacy, Yonsei University, Incheon 21983, Republic of Korea
}

Received March 20, 2017; Accepted October 6, 2017

DOI: $10.3892 /$ ijmm.2017.3184

\begin{abstract}
Polycyclic aromatic hydrocarbons (PAHs) are widespread contaminants resulting from the incomplete combustion of organic materials in the environment. The primary concern for the hazardous effect of PAHs is their ability to activate the pathway linked to the aryl hydrocarbon receptor (AhR) and lead to carcinogenesis. While previous research has demonstrated that oxidative stress plays a key role in the AhR-dependent toxic response, the effect of PAHs on the biosynthesis of glutathione (GSH), which is a powerful endogenous antioxidant, has not been extensively investigated. In the present study, we utilized a global metabolomic approach, via high resolution magic angle spinning nuclear magnetic resonance spectroscopy, and identified significant metabolome differences between non-tumorigenic liver cells (BNL CL.2; CL2) and transformed liver cells (BNL 1MEA. 7R.1; 1MEA) chronically exposed to 3-methylcholanthrene (3MC), a well-known carcinogenic PAH. A significant change that was observed, was a lower GSH level in 1MEA cells compared with that in CL2 cells. This was contrasted by increased levels of precursor metabolites for GSH synthesis, such as $S$-adenosylmethionine and cysteine. These changes were accompanied by a significantly reduced expression of $\gamma$-glutamylcysteine ligase (GCL), known to be the rate-limiting step of GSH synthesis. Furthermore, the protein level of cysteine dioxygenase was downregulated; however, the
\end{abstract}

Correspondence to: Professor Young-Suk Jung, College of Pharmacy, Pusan National University, Busandaehag-ro, 63 Beon-gil 2, Geumjeong-gu, Busan 46241, Republic of Korea

E-mail: youngjung@pusan.ac.kr

Professor Suhkmann Kim, Department of Chemistry, Center for Proteome Biophysics and Chemistry Institute for Functional Materials, Pusan National University, Busandaehag-ro, 63 Beon-gil 2, Geumjeong-gu, Busan 46241, Republic of Korea

E-mail: suhkmann@pusan.ac.kr

*Contributed equally

Keywords: polycyclic aromatic hydrocarbon, 3-methylcholanthrene, oxidative stress, glutathione, metabolomics concentration of taurine was unaltered. Therefore, the present study demonstrated that cells transformed by chronic exposure to 3MC exhibited inhibition of GSH biosynthesis by suppression of GCL protein expression and reduction of cysteine availability, which may subsequently render cells vulnerable to oxidative stress.

\section{Introduction}

Polycyclic aromatic hydrocarbons (PAHs) are well-known air pollutants released from the incomplete combustion of wood, coal, diesel, fat and tobacco from natural as well as anthropogenic sources $(1,2)$. Long-term exposure to these chemicals has toxic effects on humans and animals, including developmental changes, abnormal immune response and carcinogenesis (3-6). The main mechanism responsible for the toxicity of PAHs is considered to be their ability to activate the aryl hydrocarbon receptor (AhR)-mediated pathway (7). Dimerization of the PAHs/AhR complex with the AhR nuclear translocator causes it to bind to its cognate DNA-binding site, the xenobiotic response element, which leads to the transcriptional activation of xenobiotic-metabolizing enzymes (XMEs) such as cytochrome P450 (CYP), epoxide hydrolase (EH), glutathione S-transferase, UDP-glucuronosyltransferase, sulfotransferase, NAD(P)H quinone oxidoreductase, and aldo-keto reductase $(8,9)$. Activated XMEs play a major role, not only in the detoxification of PAHs, but also in the conversion of PAHs into active metabolites. For example, the CYP1A1 and 1B1 enzymes, in cooperation with EH, play major roles in activating PAHs to highly reactive diol-epoxides that are considered to be carcinogenic metabolites (10).

Exposure to xenobiotics, including PAHs, may produce reactive metabolites and cause imbalance between endogenous and exogenous oxidants. This may subsequently induce a decrease in antioxidant defenses and allow oxidative tissue damage. It has been previously reported that the blood level of phenanthrene, an aromatic hydrocarbon, is positively correlated with blood malondialdehyde levels in human samples (11). Moreover, exposure of zebrafish to PAHs induced oxidative stress involved in the regulation of Nrf2 (12). In lymphoma cells, PAHs depleted intracellular levels of glutathione (GSH), contributing to cell injury (13). Moreover, chronic exposure 
to benzo(a)pyrene produced reactive oxygen species, which was accompanied by caspase- 3 activation and apoptotic cell death (14). Taken together, the findings of those studies suggest that the toxic effect of PAHs likely occurs through the oxidative stress pathway.

In the present study, metabolite profiling by global metabolomics revealed that cells chronically exposed to 3-methylcholanthrene (3MC) have low concentrations of GSH, which is a powerful endogenous antioxidant. While previous studies have demonstrated that oxidative stress plays a critical role in the AhR-dependent toxic response, the effect of PAHs on GSH biosynthesis has not been extensively investigated. GSH is a tripeptide synthesized as the final product in the transsulfuration pathway. GSH is the most abundant molecule among endogenous antioxidants and it plays a central role in sulfhydryl homeostasis, acting as a major cellular antioxidant. The concentration of GSH in cells is tightly regulated by de novo synthesis, utilization and export. However, it has been demonstrated that intracellular GSH decreases under conditions of oxidative stress, or through generation of reactive metabolites from xenobiotics (15). Reduced levels of GSH are also observed in various pathophysiological conditions, such as sepsis, acute Wilson's disease, and inherited deficiencies in GSH synthesis $(15,16)$.

The objective of the present study was to determine the effect of chronic exposure to $3 \mathrm{MC}$, a major $\mathrm{PAH}$, on the transsulfuration pathway using targeted metabolomics analysis, aiming to provide novel insights into the mechanism through which $3 \mathrm{MC}$ affects intracellular GSH levels and renders cells vulnerable to a second insult of oxidative stress.

\section{Materials and methods}

Reagents and antibodies.3MC,3-(4,5-dimethylthiazol)-2,5-diphenyltetrazolium bromide (MTT), $S$-adenosylmethionine (SAM), $S$-adenosylhomocysteine $(\mathrm{SAH}), \mathrm{L}$-amino acids analytical standard, hypotaurine, taurine, $o$-phthaldiadehyde, 7-benzo-2-oxa-1,3-diazole-4-sulfonic acid (SBD-F), tris(2-carboxyethyl)phosphine hydrochloride (TCEP), N-(2-mercaptopropionyl)glycine (MPG), GSH, tertbutyl hydroperoxide ( $t$-BHP) and crystal violet were purchased from Sigma Aldrich; Merck KGaA (St. Louis, MO, USA). Anti-cysteine dioxygenase (CDO) antibody was obtained from Abcam (Cambridge, MA, USA). Anti- $\gamma$-glutamylcysteine ligase catalytic subunit (GCL) antibody and anti-glyceraldehyde 3-phosphate dehydrogenase (GAPDH) antibody were purchased from Santa Cruz Biotechnology, Inc. (Santa Cruz, CA, USA). Peroxidase-conjugated antibodies against mouse or rabbit $\mathrm{IgG}$ were obtained from Thermo Fisher Scientific (Waltham, MA, USA).

Cell culture. The non-tumorigenic mouse liver cell line BNL CL.2 (designated as CL2) and 3MC-transformed BNL 1MEA. 7R.1 cells (designated as 1MEA), which were derived from CL2 cells chronically treated with $3 \mathrm{MC}$, were obtained from the Korea Research Institute of Bioscience and Biotechnology (Daejeon, Korea). Both cell lines were established and characterized by Gold et al (17). The cells were grown in Dulbecco's modified Eagle's medium containing $10 \%$ fetal bovine serum, $100 \mathrm{U} / \mathrm{ml}$ penicillin and $100 \mu \mathrm{g} / \mathrm{ml}$ streptomycin (GenDEPOT, Barker, TX, USA) at $37^{\circ} \mathrm{C}$ in a humidified incubator with $5 \% \mathrm{CO}_{2}$.
Metabolomic analysis using nuclear magnetic resonance (NMR) spectroscopy. A cell pellet of $25 \mathrm{mg}$ was transferred to a 4-mm NMR nanotube (Agilent Technologies, Santa Clara, CA, USA). $\mathrm{D}_{2} \mathrm{O}(25 \mu \mathrm{l})$ containing $2 \mathrm{mM} 3$-(trimethylsilyl) propionic-2,2,3,3- $\mathrm{d}_{4}$ acid sodium salt $\left(\mathrm{TSP}-\mathrm{d}_{4}\right)$ was then transferred to a separate NMR nanotube. Samples were measured using high-resolution magic angle spinning (HR-MAS) NMR spectroscopy. All spectra were acquired with a 600.167-MHz Agilent NMR spectrometer equipped with a 4-mm gHX NanoProbe (Agilent Technologies) and a spinning rate of 2,050 Hz. The Carr-Purcell-Meiboom-Gill (CPMG) pulse sequence was used due to the suppression of water and the presence of high molecular mass compounds. The acquisition time was $1.703 \mathrm{msec}$, the relaxation delay was $1 \mathrm{sec}$, and 128 transients were collected. All spectra were processed and assigned using Chenomx NMR Suite 7.1 professional with the Chenomx $600 \mathrm{MHz}$ library database (Chenomx, Edmonton, $\mathrm{AB}$, Canada). Spectra were binned from 0.5 to $10 \mathrm{ppm}$ for multivariate statistical analysis. The binning size was 0.001 ppm and water peak (4.5-4.9 ppm) and spinning side band peak were excluded $(1.15-1.2,3.61-2.69,6.56-7.16$ and 8.0-8.4 ppm). The binning data were normalized to total area. Principal component analysis (PCA) was conducted with the SIMCA-P ${ }^{+}$software package (version 12.0; Umetrics, Umeå, Sweden). $\mathrm{R}^{2}$ parameter indicates the explained variation in the data and goodness of fit and $\mathrm{Q}^{2}$ parameter represents the predictive power of the model (18).

Validation of metabolites in the transsulfuration pathway. Washed cells in 100-mm culture plates were harvested $24 \mathrm{~h}$ after a medium change by scraping in either $5 \%$ perchloric acid to determine SAM, SAH, cysteine and GSH, or in ice-cold methanol to determine the levels of methionine, glutamate, glycine, hypotaurine and taurine. The protein pellet was dissolved in $0.1 \mathrm{M} \mathrm{NaOH}$ solution, and the protein concentration was determined using a bicinchoninic acid (BCA) protein assay kit (Thermo Fisher Scientific).

SAM and SAH were analyzed by high performance liquid chromatography (HPLC) (Ultimate $3000^{\mathrm{TM}}$; Thermo Fisher Scientific) equipped with a UV detector (UV-3000; $254 \mathrm{~nm}$; Thermo Fisher Scientific), using the modified method of She et al (19). Separation was accomplished using a Hector T-C18 column (5 $\mu \mathrm{m}$ x 4.6 mm x $100 \mathrm{~mm}$ ) (Chiral Technology Korea, Daejeon, Korea).

Methione, glutamate, glycine, hypotaurine and taurine were derivatized with $o$-phthalaldehyde/2-mercaptoethanol and quantified using HPLC with a fluorescence detector (FLD-3100; excitation wavelength, $338 \mathrm{~nm}$; and emission wavelength, $425 \mathrm{~nm}$; Thermo Fisher Scientific) $(20,21)$. They were separated using a Hector T-C18 column (3 $\mu \mathrm{m}$ x $4.6 \mathrm{~mm}$ x $100 \mathrm{~mm}$ ).

Total cysteine and GSH were analyzed using the SBD-F sample derivatization method (22). MPG (50 $\mu \mathrm{l})$, as an internal standard, was added to the samples $(50 \mu \mathrm{l})$ and briefly mixed by vortex. Following the addition of $10 \mu \mathrm{l}$ of a $10 \%$ (w/v) TCEP solution, the tubes were incubated at room temperature for $30 \mathrm{~min}$. Subsequently, $90 \mu \mathrm{l}$ of a $10 \%$ (w/v) trichloroacetic acid solution with $1 \mathrm{mM}$ ethylenediaminetetraacetic acid (EDTA) was added to each sample, briefly mixed by vortex and centrifuged $(13,000 \mathrm{x} \mathrm{g}$ for $10 \mathrm{~min})$. The supernatant $(50 \mu \mathrm{l})$ was then 
added to another tube containing $10 \mu \mathrm{l}$ of $1.55 \mathrm{M} \mathrm{NaOH}, 125 \mu \mathrm{l}$ of $0.125 \mathrm{M}$ borate buffer ( $\mathrm{pH} 9.5$ ) with $4 \mathrm{mM}$ EDTA, and $50 \mu \mathrm{l}$ of $0.1 \%$ (w/v) SBD-F in borate buffer (0.125 M with 4 mM EDTA). The samples were incubated at $60^{\circ} \mathrm{C}$ for $1 \mathrm{~h}$, and a $20 \mu \mathrm{l}$ aliquot was then injected into the HPLC equipped with a fluorescence detector (excitation wavelength, $385 \mathrm{~nm}$; and emission wavelength, $515 \mathrm{~nm})$. The chromatographic separation was achieved using a Hector M-C18 column (3 $\mu \mathrm{m}$ x $4.6 \mathrm{~mm}$ x $150 \mathrm{~mm})$.

Immunoblot analysis. Cells were lysed with ice-cold PRO-PREP ${ }^{\text {TM }}$ protein extract solution (Intron, Seongnam, Korea), and the protein concentration was quantified using the BCA procedure (Thermo Fisher Scientific). Equal amounts of protein samples were separated by sodium dodecyl sulfate-polyacrylamide gel electrophoresis using a 10\% polyacrylamide gel, and then transferred onto a polyvinylidene difluoride membrane (Millipore, Billerica, MA, USA). The membrane was blocked with $5 \%$ skimmed milk in $100 \mathrm{mM}$ Tris- $\mathrm{HCl}$ (pH 7.5), $150 \mathrm{mM} \mathrm{NaCl}$ and $0.2 \%$ Tween-20 (TBST) for $1 \mathrm{~h}$ at room temperature. The blots were incubated overnight with primary antibodies diluted in TBST containing 5\% milk at $4^{\circ} \mathrm{C}$. After three washes with TBST, the blot was incubated with the appropriate horseradish peroxidase-conjugated secondary antibodies. The antigen was detected using a Western Bright ECL HRP substrate kit (Advansta, Menlo Park, CA, USA).

Determination of cell viability. Cells were plated in a 96-well plate and cell viability was determined $24 \mathrm{~h}$ after $t$-BHP treatment by the MTT assay, according to the manufacturer's instructions. Briefly, after incubation with MTT $(0.5 \mathrm{mg} / \mathrm{ml})$ for $4 \mathrm{~h}$ at $37^{\circ} \mathrm{C}$, formazan precipitates formed by mitochondrial dehydrogenases in viable cells were extracted with dimethyl sulfoxide. The absorbance of the converted dye was measured at $540 \mathrm{~nm}$ using the Multiskan GO reader (Thermo Fisher Scientific), and the results were expressed as a percentage (\%) compared with the vehicle treatment.

Fluorescence-activated cell sorting (FACS) analysis. Apoptotic and live cells were determined by FACS analysis using Annexin V-FITC staining. The cells were treated with $t$-BHP for $24 \mathrm{~h}$, and were subsequently harvested, trypsinized, washed once in cold phosphate-buffered saline and suspended in $1 \mathrm{X}$ binding buffer. The counted cells were stained in propidium iodide and Annexin V-FITC solution (Annexin V-FITC apoptosis detection kit; BD Biosciences, Bedford, MA, USA) at room temperature for $15 \mathrm{~min}$ in the dark. The stained cells were analyzed by flow cytometry within $1 \mathrm{~h}$. Apoptotic and live cells were analyzed by Becton Dickinson FACSscan flow cytometer and BD FACSDiva software (BD Biosciences).

Statistical analysis. All the results are expressed as mean \pm standard deviation $(n=5)$ and were analyzed by a two-tailed Student's t-test. The acceptable level of statistical significance was set at $\mathrm{P}<0.05$.

\section{Results}

Metabolite profiling and PCA of ${ }^{I} H$ NMR spectroscopy in CL2 and 1MEA cells. Upon ${ }^{1} \mathrm{H}$ NMR analysis, pattern recognition using PCA of the NMR spectra in metabolite profiling revealed clear clustering between CL2 and 1MEA cells (Fig. 1A). The PCA loading plots revealed that several metabolites, such as acetate, alanine, creatine, glutamate, glutamine, glycine, sn-glycero-3-phosphocholine, GSH, myo-inositol, lactate and $o$-phosphocholine, differed significantly between the two cell types (Fig. 1B). In detailed spectra analysis, it was identified that acetate $(\mathrm{x} 0.21)$, choline $(\mathrm{x} 0.72)$, formate $(\mathrm{x} 0.51), \mathrm{GSH}$ (x0.59), glycerol (x0.38), isoleucine (x0.61), leucine $(\mathrm{x} 0.62)$, niacinamide (x0.44), $o$-phosphocholine (x0.06), phenylalanine (x0.63), serine (x0.71), succinate $(\mathrm{x} 0.56)$, tyrosine $(\mathrm{x} 0.68)$, uridine (x0.42), valine (x0.70) and sn-glycero-3-phosphocholine $(\mathrm{x} 0.31)$ were significantly lower in the 1MEA cells compared with the CL2 cells $(\mathrm{P}<0.05)$. Alternatively, glutamate (x1.61), glutamine (x6.17), glycine (x1.45), proline (x2.02) and myo-inositol (x1.32) were significantly higher in 1MEA cells compared with CL2 cells ( $\mathrm{P}<0.05$; Fig. 1C).

1MEA cells exhibit aberrant changes in metabolites of the transsulfuration pathway. GSH plays a critical role in removing reactive metabolites from the cell, and a disturbance in its synthesis reduces a cell's resistance to oxidative stress. Since there are limited data available showing that $3 \mathrm{MC}$ causes oxidative stress, attention was paid to the significantly lower level of intracellular GSH in 1MEA cells compared with that in CL2 cells. Specifically, we focused on the transsulfuration pathway that produces GSH and taurine from the essential amino acid methionine (Fig. 2). To investigate and validate all the major metabolites in the transsulfuation pathway, HPLC with a UV or fluorescence detector was used. The cellular levels of SAM, cysteine, glutamate and glycine were significantly increased 2.0-, 2.7-, 2.1- and 1.4-fold, respectively, in 1MEA cells compared with CL2 cells (Fig. 3B, D-F). On the contrary, the concentration of SAH was slightly decreased in 1MEA cells (Fig. 3C). The levels of methionine, hypotaurine and taurine did not change (Fig. 3A, H and I), but the GSH levels in 1MEA cells were significantly reduced compared with those in CL2 cells (Fig. 3G). Cysteine is the precursor metabolite used to synthesize both GSH and taurine, with GSH being a tripeptide composed of glutamate, cysteine and glycine. Considering the increased levels of cysteine, glutamate and glycine in 1MEA cells, the decreased levels of GSH cannot be fully explained. To investigate why the GSH levels decreased in 1MEA cells, the expression of CDO and GCL, which are the rate-limiting enzymes in the production of taurine and GSH, respectively, was subsequently investigated. In 1MEA cells, CDO expression decreased to $60 \%$ of that in CL2 cells, whereas GCL expression decreased to $11 \%$ of that in CL2 cells (Fig. 4). Thus, it is suggested that reduced GCL protein expression contributes to decreased GSH synthesis.

1MEA cells are more vulnerable to cell death incuced by t-BHP treatment compared with CL2 cells. To investigate whether low concentrations of GSH in 1MEA cells affected the rate of cell death by exogenous oxidative stress, the cells were exposed to $t$-BHP, a producer of reactive oxygen species, with cell viability being examined $24 \mathrm{~h}$ post-treatment (Fig. 5). As expected, 1MEA cells were more sensitive to $t$-BHP treatment, with a $50 \mu \mathrm{M} t$-BHP treatment leading to $50 \% 1 \mathrm{MEA}$ cell death rate, while the survival of CL2 cells was not affected. To determine oxidative stress-induced 
A

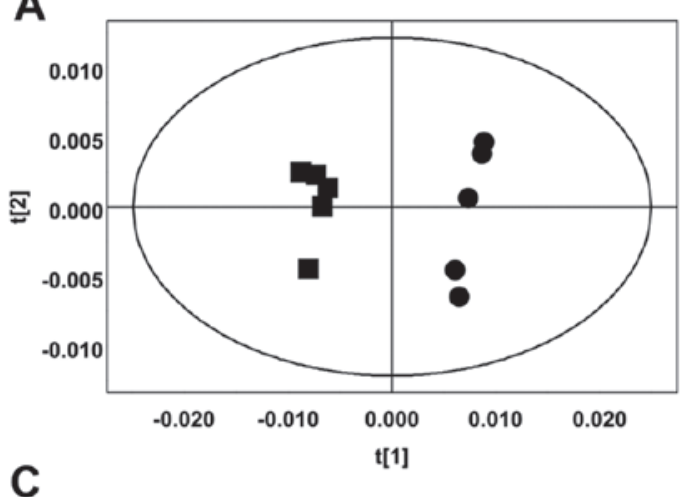

B

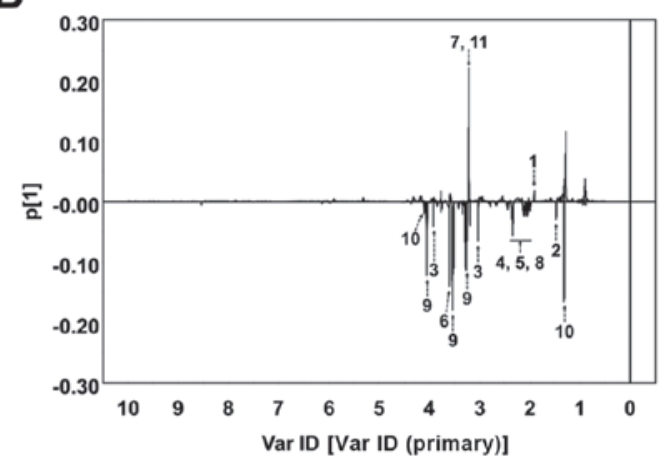

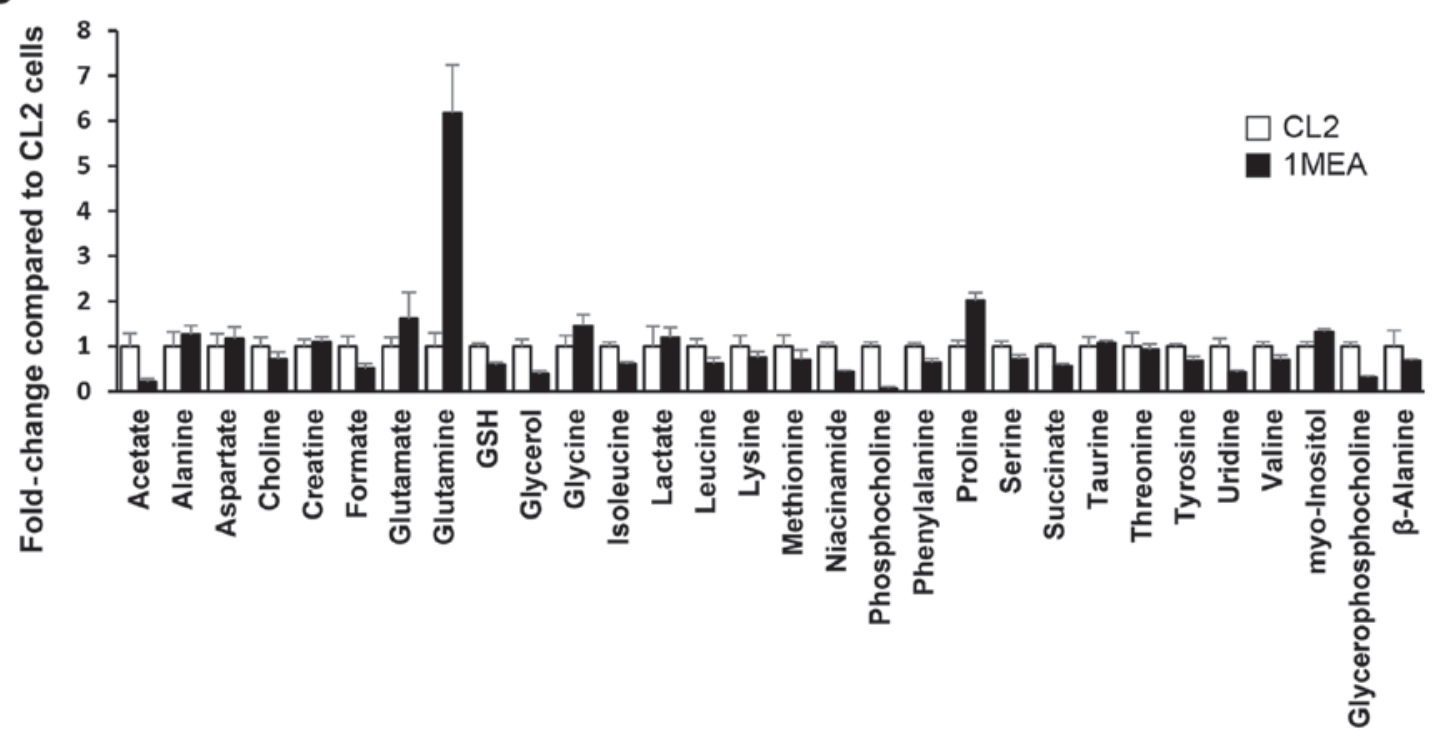

Figure 1. Results of ${ }^{1} \mathrm{H}$ nuclear magnetic resonance (NMR) spectroscopy in CL2 and $1 \mathrm{MEA}$ cells. $\left(\mathrm{R}^{2} \mathrm{X}=0.873, \mathrm{Q}^{2}=0.682\right)$ (A) Principal component analysis (PCA) score plot. Circles, CL2; squares, 1MEA. (B) PCA loading plot of CL2 vs. 1MEA. 1, Acetate; 2, alanine; 3, creatine; 4, glutamate; 5, glutamine; 6, glycine; 7, glycerophosphocholine; 8, GSH; 9, inositol; 10, lactate; 11, phosphocholine. (C) Fold changes of quantified metabolites from ${ }^{1} \mathrm{H}$ NMR spectra. Each value represents the mean \pm standard deviation. GSH, glutathione.

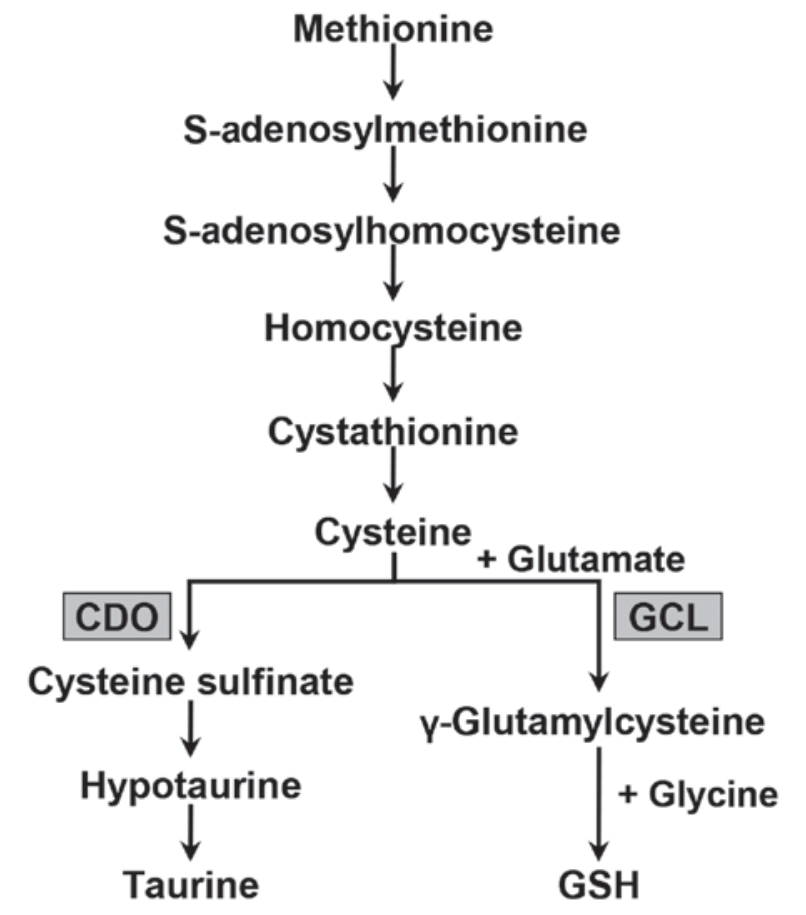

Figure 2. Metabolic pathway of transsulfuration. CDO, cysteine dioxygenase; GCL, $\gamma$-glutamylcysteine ligase; GSH, glutathione. apoptotic cell death, flow cytometry analysis was performed. As shown in Fig. 6, increase of early apoptosis (lower right quadrant) and late apoptosis (upper right quadrant) were clearly observed in $t$-BHP-treated 1MEA cells compared with CL2 cells.

\section{Discussion}

There is increasing evidence that GSH is reduced in several human diseases, and this contributes to the deterioration of the condition (23). In a number of of these diseases, GSH depletion, which is often caused by decreases in the expression of GSH synthesizing enzymes, is considered to play a major role in oxidative tissue damage (24). In the present study, 1MEA cells, transformed by chronically exposing CL2 cells to $3 \mathrm{MC}$, were found to be more susceptible to oxidative stress compared with CL2 cells, due to the decreased synthesis of GSH. Although the main concern regarding PAH toxicity is its ability to activate the AhR-mediated pathway and lead to cancer development, these results indicate that the deleterious effect of $3 \mathrm{MC}$ may be mediated by a reduction in the antioxidant capacity of the cells.

To understand the altered metabolism and identify abnormal pathways in response to chronic 3MC exposure, we 
A

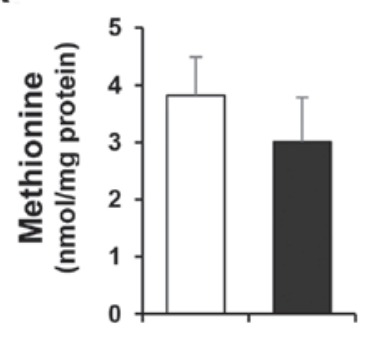

D

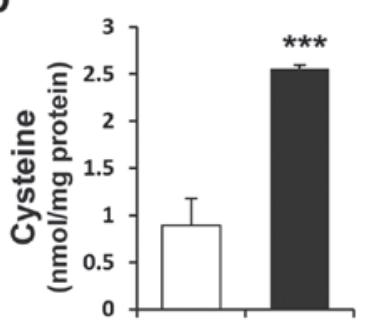

G

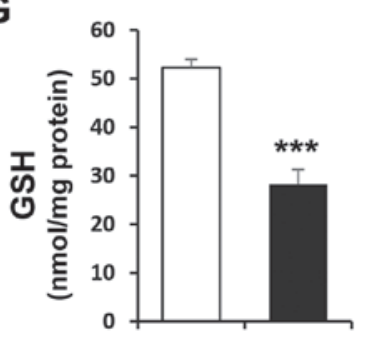

B

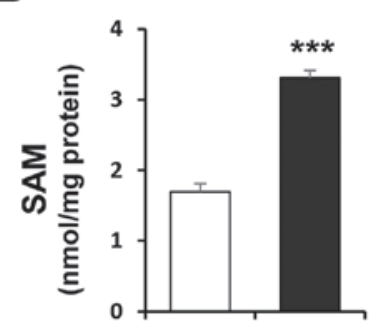

$\mathbf{E}$

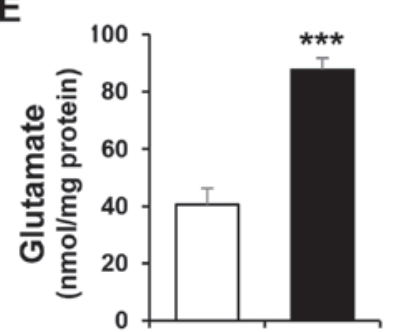

H

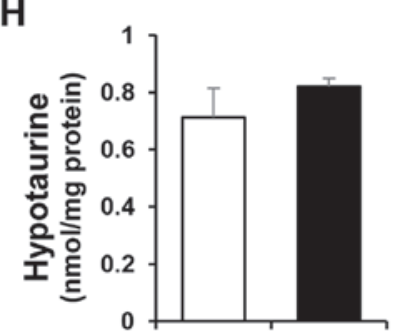

C

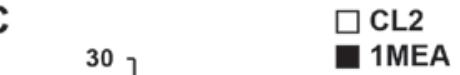

$\mathbf{F}$

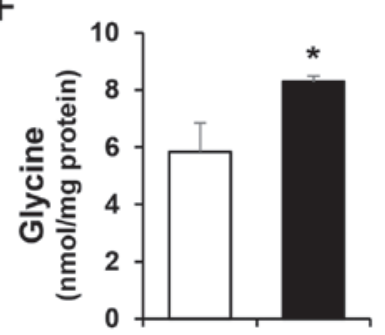

I

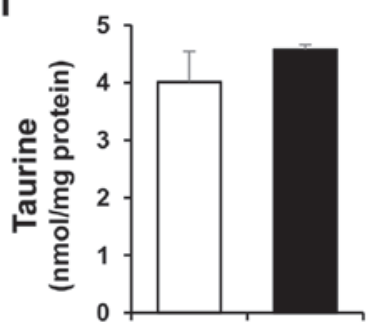

Figure 3. Cellular levels of sulfur amino acids and their metabolites in CL2 and 1MEA cells. (A) Methionine, (B) S-adenosylmethionine (SAM); (C) S-adenosylhomocysteine (SAH); (D) cysteine; (E) glutamate; (F) glycine; (G) glutathione (GSH); (H) hypotaurine, (I) taurine. Each value represents the mean \pm standard deviation. The differences between CL2 and 1MEA cells were statistically significant $\left({ }^{*} \mathrm{P}<0.05\right.$ and ${ }^{* * * *} \mathrm{P}<0.001 ;$ Student's t-test).
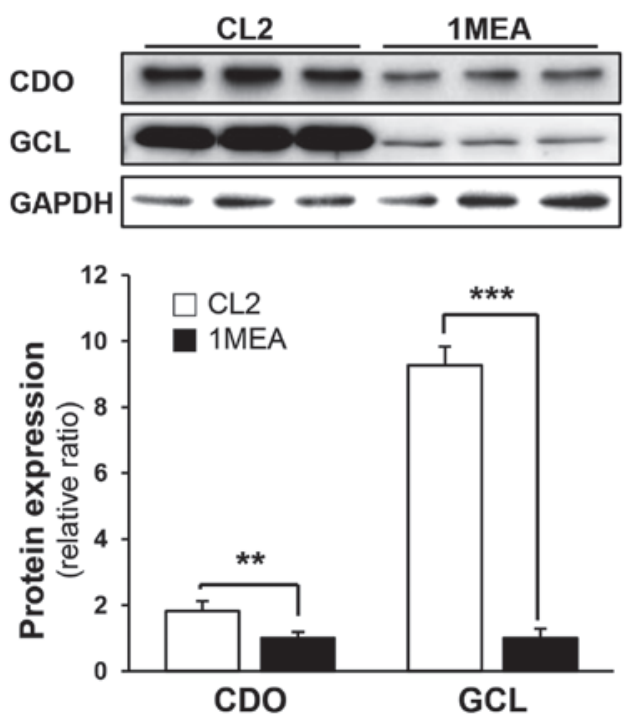

Figure 4. Protein expression of cysteine dioxygenase (CDO) and $\gamma$-glutamylcysteine ligase (GCL) and viability of CL2 and 1MEA cells. Blots of CDO and GCL in CL2 and 1MEA cells resolved by immunoblot analysis were quantified densitometrically. Each value represents the mean \pm standard deviation. The differences between CL2 and 1MEA cells were statistically significant $\left({ }^{* * *} \mathrm{P}<0.01\right.$ and ${ }^{* * *} \mathrm{P}<0.001$; Student's t-test).

have introduced the technique of global metabolite profiling followed by targeted metabolomic analysis. Specifically, HR-MAS NMR spectroscopy was used to perform

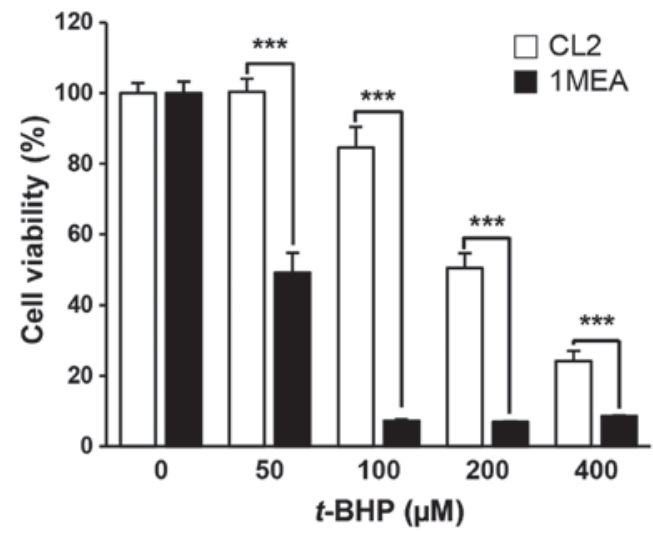

Figure 5. Effect of tert-butyl hydroperoxide ( $t$-BHP) treatment on the viability of CL2 and 1MEA cells. The cells were treated with indicated concentrations of $t$-BHP for $24 \mathrm{~h}$, followed by an MTT assay to determine viability. Each value represents the mean \pm standard deviation. The differences between CL2 and 1MEA cells were statistically significant $\left({ }^{* * * *} \mathrm{P}<0.001\right.$; Student's t-test).

non-destructive metabolite profiling of cells. Thus, changes in the metabolites involved in the synthesis of sulfur-containing amino acids, which are associated with a cellular redox state, were observed.

Sulfur-containing amino acid metabolism occurs primarily via the transsulfuration pathway, which results in the transfer of the methionine sulfur to serine to form cysteine (Fig. 2). Subsequently, cysteine is irreversibly metabolized to yield 
A
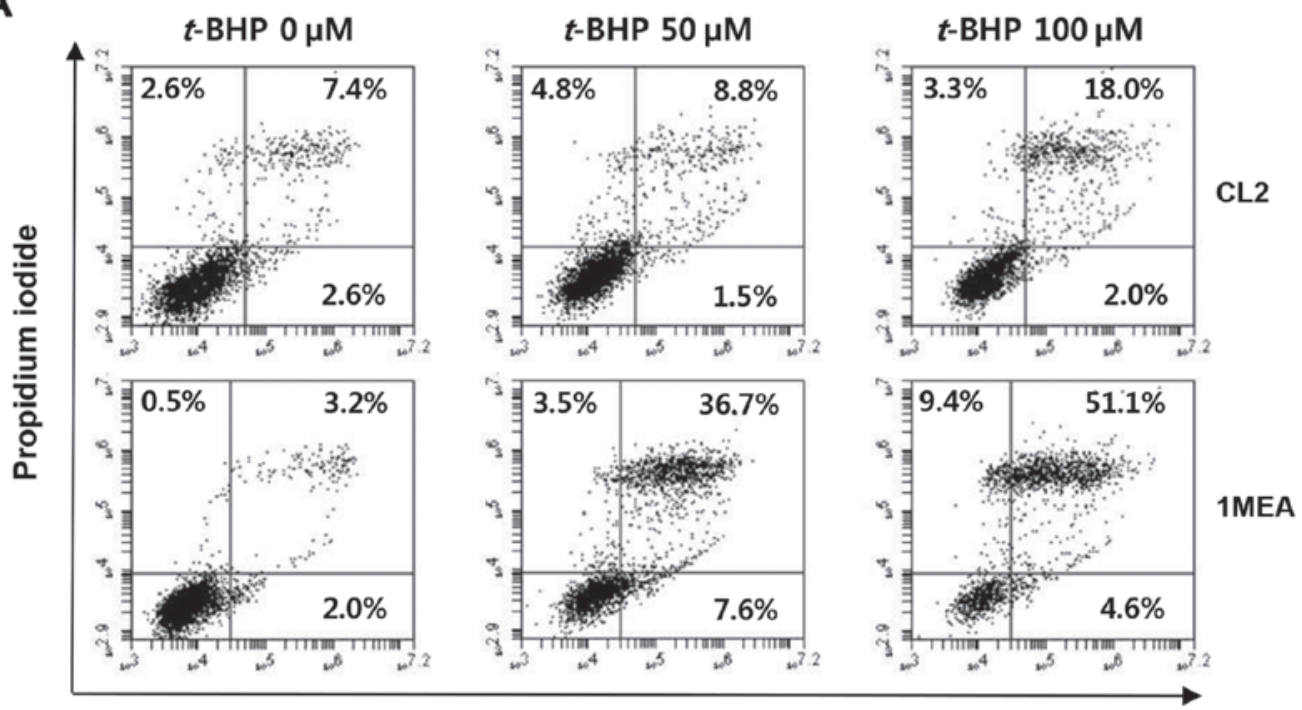

Annexin V-FITC

B

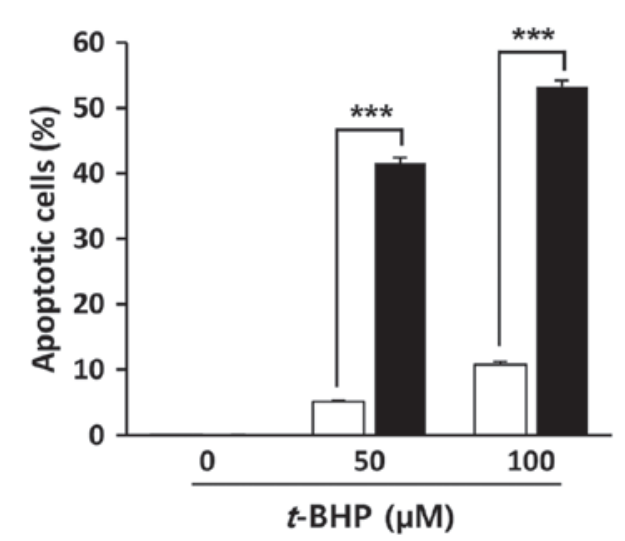

C

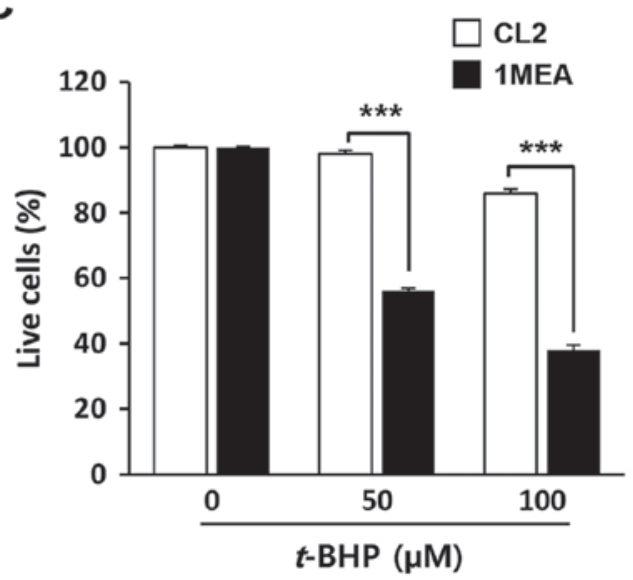

Figure 6. Quantification of tert-butyl hydroperoxide ( $t$-BHP)-induced apoptosis in CL2 and 1MEA cells. (A) The cells were treated with the indicated concentrations of $t$-BHP for $24 \mathrm{~h}$, followed by a fluorescence-activated cell sorting analysis after propidium iodide uptake and Annexin V binding in non-permeabilized cells (lower left, live cells; lower right, early apoptotic cells; upper right, late apoptotic cells). Quantification of (B) apoptotic cells and (C) live cells was represented in the three independent experiments. Each value represents the mean \pm standard deviation. The differences between CL2 and $1 \mathrm{MEA}$ cells were statistically significant $\left({ }^{* * *} \mathrm{P}<0.001\right.$; Student's t-test). FITC, fluorescein isothiocyanate.

taurine or GSH. CDO catalyzes the oxidation of cysteine to cysteine sulfinate, which is converted primarily to taurine via hypotaurine and the enzyme cysteine sulfinate decarboxylase. Alternatively, the synthesis of GSH from its constituent amino acids involves two ATP-requiring enzymatic steps: The first step of GSH biosynthesis is rate-limiting and catalyzed by GCL to make $\gamma$-glutamylcysteine from glutamate and cysteine; the second step in GSH synthesis is catalyzed by GSH synthetase to form GSH from $\gamma$-glutamylcysteine and glycine (25).

The increase in the amount of amino acids that constitute GSH, and the seemingly contradictory decrease in the concentration of GSH in 1MEA cells, as compared to CL2 cells, were of particular interest in the present study. Synthesis of GSH is largely limited by two factors: The availability of cysteine and the activity of GCL (26). Cysteine concentrations are regulated by a balance between the rates of its synthesis through the transsulfuration pathway and its metabolism to GSH, inorganic sulfate, or taurine $(27,28)$. Previous studies have suggested that cysteine availability is a major determining factor for the parti- tioning of cysteine sulfur to either GSH, taurine, or inorganic sulfates in rat liver $(29,30)$. Specifically, low cysteine availability would favor its utilization for the synthesis of GSH, and high cysteine availability enhances its catabolism to inorganic sulfate and taurine. Although CDO expression in 1MEA cells was lower compared with that in CL2 cells, it did not lead to any difference in hypotaurine and taurine concentrations between groups, thereby indicating that a sufficient amount of cysteine is supplied to synthesize taurine. However, GCL expression in 1MEA cells was markedly reduced, which may account for the increased cysteine concentrations, as well as the reduced GSH levels. These results suggest that blocking GSH synthesis may have a major effect on the partitioning of cysteine sulfur to taurine synthesis.

In conclusion, the present results indicate that cells transformed by chronic exposure to 3MC exhibited abnormal changes in the transsulfuration pathway, accompanied by increased availability of cysteine to taurine rather than GSH synthesis. Inhibition of cysteine catabolism to GSH by $3 \mathrm{MC}$ may play an important role in the preservation of intracellular 
taurine. Finally, the potentiation of $t$-BHP-induced death in 1MEA cells appears to be associated with a blockage in GSH synthesis. It has been reported that long-term exposure to PAHs, including $3 \mathrm{MC}$, is the cause of serious health problems by various cellular effects. Further studies to determine the biological effect of $3 \mathrm{MC}$ on the dysregulation of the transsulfuration pathway are currently underway in this laboratory.

\section{Acknowledgements}

This study was supported by the National Research Foundation of Korea (NRF) grant funded by the Korean government (MSIP) (no. 2009-0083538). The study was also supported by the Basic Science Research Program through the National Research Foundation of Korea (NRF) funded by the Ministry of Science, ICT and Future Planning (grant no. NRF-2014R1A 1A1005435).

\section{References}

1. Van Metre PC and Mahler BJ: Trends in hydrophobic organic contaminants in urban and reference lake sediments across the United States, 1970-2001. Environ Sci Technol 39: 5567-5574, 2005.

2. Weisman D, Alkio M and Colón-Carmona A: Transcriptional responses to polycyclic aromatic hydrocarbon-induced stress in Arabidopsis thaliana reveal the involvement of hormone and defense signaling pathways. BMC Plant Biol 10: 59, 2010.

3. Kim KH, Jahan SA, Kabir E and Brown RJ: A review of airborne polycyclic aromatic hydrocarbons (PAHs) and their human health effects. Environ Int 60: 71-80, 2013.

4. Pelclová D, Fenclová Z, Dlasková Z, Urban P, Lukás E, Procházka B, Rappe C, Preiss J, Kocan A and Vejlupková J: Biochemical, neuropsychological, and neurological abnormalities following 2,3,7,8-tetrachlorodibenzo-p-dioxin (TCDD) exposure. Arch Environ Health 56: 493-500, 2001.

5. Baccarelli A, Mocarelli P, Patterson DG Jr, Bonzini M, Pesatori AC, Caporaso N and Landi MT: Immunologic effects of dioxin: New results from Seveso and comparison with other studies. Environ Health Perspect 110: 1169-1173, 2002.

6. Pesatori AC, Consonni D, Bachetti S, Zocchetti C, Bonzini M, Baccarelli A and Bertazzi PA: Short- and long-term morbidity and mortality in the population exposed to dioxin after the 'Seveso accident'. Ind Health 41: 127-138, 2003.

7. Mimura $J$ and Fujii-Kuriyama Y: Functional role of AhR in the expression of toxic effects by TCDD. Biochim Biophys Acta 1619: 263-268, 2003.

8. Denison MS and Nagy SR: Activation of the aryl hydrocarbon receptor by structurally diverse exogenous and endogenous chemicals. Annu Rev Pharmacol Toxicol 43: 309-334, 2003.

9. Nebert DW, Dalton TP, Okey AB and Gonzalez FJ: Role of aryl hydrocarbon receptor-mediated induction of the CYP1 enzymes in environmental toxicity and cancer. J Biol Chem 279: 23847-23850, 2004.

10. Shimada T: Xenobiotic-metabolizing enzymes involved in activation and detoxification of carcinogenic polycyclic aromatic hydrocarbons. Drug Metab Pharmacokinet 21: 257-276, 2006.

11. Suresh R, Shally A, Mahdi AA, Patel DK, Singh VK and Rita M: Assessment of association of exposure to polycyclic aromatic hydrocarbons with bronchial asthma and oxidative stress in children: A case control study. Indian J Occup Environ Med 13: 33-37, 2009.
12. Van Tiem LA and Di Giulio RT: AHR2 knockdown prevents PAH-mediated cardiac toxicity and XRE- and ARE-associated gene induction in zebrafish (Danio rerio). Toxicol Appl Pharmacol 254: 280-287, 2011.

13. Yuan JW, Krieger JA, Maples KR, Born JL and Burchiel SW: Polycyclic aromatic hydrocarbons decrease intracellular glutathione levels in the A20.1 murine B cell lymphoma. Fundam Appl Toxicol 23: 336-341, 1994.

14. Ranjit S, Midde NM, Sinha N, Patters BJ, Rahman MA, Cory TJ, Rao PS and Kumar S: Effect of polyaryl hydrocarbons on cytotoxicity in monocytic cells: Potential role of cytochromes P450 and oxidative stress pathways. PLoS One 11: e0163827, 2016.

15. Lu SC: Glutathione synthesis. Biochim Biophys Acta 1830: 3143-3153, 2013

16. Summer KH and Eisenburg J: Low content of hepatic reduced glutathione in patients with Wilson's disease. Biochem Med 34: 107-111, 1985.

17. Gold LS, Slone TH, Manley NB and Bernstein L: Target organs in chronic bioassays of 533 chemical carcinogens. Environ Health Perspect 93: 233-246, 1991.

18. Wheelock AM and Wheelock CE: Trials and tribulations of 'omics data analysis: Assessing quality of SIMCA-based multivariate models using examples from pulmonary medicine. Mol Biosyst 9: 2589-2596, 2013

19. She QB, Nagao I, Hayakawa T and Tsuge H: A simple HPLC method for the determination of S-adenosylmethionine and S-adenosylhomocysteine in rat tissues: The effect of vitamin B6 deficiency on these concentrations in rat liver. Biochem Biophys Res Commun 205: 1748-1754, 1994.

20. Jung YS, Kim SJ, Kwon DY and Kim YC: Comparison of the effects of buthioninesulfoximine and phorone on the metabolism of sulfur-containing amino acids in rat liver. Biochem Biophys Res Commun 368: 913-918, 2008

21. Ide T: Simple high-performance liquid chromatographic method for assaying cysteinesulfinic acid decarboxylase activity in rat tissue. J Chromatogr B Biomed Sci Appl 694: 325-332, 1997.

22. Nolin TD, McMenamin ME and Himmelfarb J: Simultaneous determination of total homocysteine, cysteine, cysteinylglycine, and glutathione in human plasma by high-performance liquid chromatography: Application to studies of oxidative stress. J Chromatogr B Analyt Technol Biomed Life Sci 852: 554-561, 2007.

23. Ballatori N, Krance SM, Notenboom S, Shi S, Tieu K and Hammond CL: Glutathione dysregulation and the etiology and progression of human diseases. Biol Chem 390: 191-214, 2009.

24. Lu SC: Regulation of glutathione synthesis. Mol Aspects Med 30: 42-59, 2009.

25. Stipanuk MH: Sulfur amino acid metabolism: Pathways for production and removal of homocysteine and cysteine. Annu Rev Nutr 24: 539-577, 2004

26. Meister A and Anderson ME: Glutathione. Annu Rev Biochem 52: 711-760, 1983.

27. Coloso RM, Drake MR and Stipanuk MH: Effect of bathocuproine disulfonate, a copper chelator, on cyst(e)ine metabolism by freshly isolated rat hepatocytes. Am J Physiol 259: E443-E450, 1990.

28. Garcia RA and Stipanuk MH: The splanchnic organs, liver and kidney have unique roles in the metabolism of sulfur amino acids and their metabolites in rats. J Nutr 122: 1693-1701, 1992.

29. Kwon YH and Stipanuk MH: Cysteine regulates expression of cysteine dioxygenase and gamma-glutamylcysteine synthetase in cultured rat hepatocytes. Am J Physiol Endocrinol Metab 280: E804-E815, 2001.

30. Stipanuk MH, Coloso RM, Garcia RA and Banks MF: Cysteine concentration regulates cysteine metabolism to glutathione, sulfate and taurine in rat hepatocytes. J Nutr 122: 420-427, 1992. 